\title{
Renal access through the inferior calyx is associated with higher risk of severe bleeding after percutaneous nephrolithotomy
}

\author{
Jing Tan, Binghai Chen, Leye He, Guangming Yin, Zhiqiang Jiang, Kun Yao, Xianzheng Jiang
}

Department of Urology, the Third Xiangya Hospital, Central South University, Changsha, China

Submitted: 18 September 2012

Accepted: 28 April 2013

Arch Med Sci 2015; 11, 2: 340-345

DOI: 10.5114/aoms.2015.50966

Copyright ๑ 2015 Termedia \& Banach

\section{Abstract}

Introduction: Hemorrhage is a major complication after percutaneous nephrolithotomy (PCNL). In the current study, we analyzed the risk factors for severe bleeding after PCNL.

Material and methods: Medical records of 982 consecutive patients undergoing PCNL at the Department of Urology, the Third Xiangya Hospital, were reviewed. The type of stone included: solitary $(n=471)$, multiple $(n=192)$ and staghorn $(n=319)$. 139 patients had renal stones of solitary kidney or functional solitary kidney. The puncture site was the inferior calyx in 246 cases, the middle calyx in 651 cases, and the upper calyx in the remaining 85 cases.

Results: The success rate (complete removal of the stone) was $92.8 \%, 73.4 \%$, and $80.9 \%$ for solitary, multiple and staghorn stones, respectively. Severe bleeding occurred in $3.25 \%(8 / 246)$ of the cases with inferior calyx access, and $0.3 \%(2 / 651)$ in cases with middle calyx access. No severe bleeding occurred in patients with upper calyx access. The bleeding was controlled successfully with focal embolization under angiography in all 10 cases.

Conclusions: Severe postoperative bleeding after PCNL is associated with renal puncture via the inferior calyx, multiple renal stones and solitary kidney stones.

Key words: nephrolithiasis, percutaneous nephrolithotomy, bleeding, embolization.

\section{Introduction}

Percutaneous nephrolithotomy (PCNL) was established as a minimally invasive treatment option for removal of kidney stones in the 1970s [1]. Today, it is the treatment of choice for large-volume stone disease with the advantages of better stone clearance, cost-effectiveness, and early convalescence compared with other modalities such as shock wave lithotripsy (SWL) or open surgery. Despite PCNL's effectiveness, serious complications such as blood loss, adjacent organ injuries, and life-threatening medical complications have been identified because of it and occur at a reported rate of up to $83 \%$ [2-5]. One of the most serious complications is renal hemorrhage. Bleeding is a significant intraoperative complication not only because of blood loss but also because it can impair vision, which may cause premature termination of the procedure

\author{
Corresponding author: \\ Xianzheng Jiang MD \\ Department of Urology \\ the Third Xiangya Hospital \\ Central South University \\ No. 138, Tongzipo Rd \\ Changsha 410013, China \\ Phone: +8673188618578 \\ Fax: +8673188618575 \\ E-mail: jiangxz123@yeah.net
}


[5]. In the literature, blood loss requiring transfusion is reported in $0.4-23 \%$ of cases and is associated with stone burden, sheath size, number of tracts, and operative time [2, 5-9]. However, previous studies with regards to risk factors have not achieved consistent conclusions [2, 5-9].

The purpose of this study was to detect the potential risk factors for severe postoperative bleeding in 982 patients with kidney stones undergoing PCNL.

\section{Material and methods}

In the current study, we retrospectively analyzed 982 consecutive patients ( 542 men and 440 women, age range: 6 to 80 years old) undergoing PCNL at the Department of Urology, the Third Xiangya Hospital, between December 2005 and December 2010. The types of renal stone included: solitary ( $n=471)$, multiple $(n=192$; average stone size $4.3 \pm 2.9 \mathrm{~cm})$ and staghorn $(n=319$ cases, average stone size $5.8 \pm 4.8 \mathrm{~cm}$ ). The location of solitary renal stones included the pelvis $(n=210 ; 2.6 \pm 2.2$ $\mathrm{cm})$, upper calyx $(n=85 ; 2.1 \pm 1.5 \mathrm{~cm})$, middle calyx $(n=124 ; 2.2 \pm 1.7 \mathrm{~cm})$, and lower calyx $(n=52$; $2.1 \pm 1.0 \mathrm{~cm}$ ). In 37 cases of multiple stones and 85 of staghorn stones, multiple access tracts were needed. The patients had solitary kidney or functional solitary kidney with contralateral atrophy (differential function less than $5 \%$ and contralateral glomerular filtration rate (GFR) $<10 \mathrm{ml} / \mathrm{min}$ ) in 139 cases. The puncture site was the inferior calyx in 246 cases, the middle calyx in 651 cases, and the upper calyx in the remaining 85 cases.

A 7 Fr catheter was placed into the urethra with a nephroscope. Direct percutaneous access to the calyx was achieved under ultrasound guidance in the prone position. The tract was dilated with a nephrostomy dilator set prior to placing an 18-20 Fr Amplatz sheath. The kidney stone was broken up with a holmium laser and removed with irrigation or forceps under a nephroscope. The nephrostomy tube was left in the pelvis for postoperative urine drainage (Figure 1). Bleeding during the surgery was managed by sealing the nephrostomy tube during the surgery, and monitored 1 week later.

Initial conservative management upon severe bleeding (hematuria that required blood transfusion of at least two units) included hemostatic drugs, antibiotics, adequate hydration and absolute bed rest. If bleeding persisted (continuing hematuria that led to a further drop in hematocrit, tachycardia and hypotension), selective renal arteriography and embolization was used to stop the bleeding. Altogether, 10 patients required selective renal arteriography and embolization. Severe postoperative bleeding in the current study was defined as bleeding that required renal arteriography and embolization.

\section{Statistical analysis}

All analyses were performed using SPSS, version 15.0 (SPSS, Inc., Chicago, IL). The $\chi^{2}$ test was used to evaluate the association between categorical variables. One-way analysis of variance and $t$-test were used to compare the means of continuous variables. A logistic model was used to determine the odds ratios for statistically significant parameters affecting severe postoperative bleeding. Data were expressed as mean \pm standard deviation. Statistical significance was considered at $p<0.05$.

\section{Results}

The overall success rate for complete stone removal was $85.1 \%$ (836/982). In patients with solitary renal stones, the success rate was $92.8 \%$ $(437 / 471)$. The success rate was considerably lower at $73.4 \%(141 / 192)$ and $80.9 \%$ (258/319) for patients with multiple stones and staghorn stones, respectively.

Severe postoperative bleeding occurred after PCNL in 10 cases. Injury to the renal arterioles was confirmed with angiography in all 10 cases. The puncture site was the inferior calyx in 8 cases $(8 / 246)$ and the middle calyx in the remaining 2 cases (2/651). Re-bleeding occurred in 2 patients within $24 \mathrm{~h}$, and was treated successfully with repeat angioembolization. Renal angioscintigraphy with technetium 99mTc-MAG3 at 3 months after PCNL was used to evaluate the renal function $[10,11]$ and showed absence of the radioisotope in target areas. No clinical evidence of postoperative infection or renal colic pain was observed.

In this study, predictive factors for severe postoperative bleeding included: stone type (solitary, multiple vs. staghorn); number of punctures; whether the patient received prior extracorporeal shock wave lithotripsy (ESWL) treatment; multiple tracts; the puncture site; solitary kidney; pre-existing urinary infection; operation time; and renal function (chronic renal failure or not).

Univariate analysis indicated that severe postoperative bleeding is correlated with lower calyx access $(p<0.001)$ and solitary kidney stones ( $p=$ 0.030 ), and closely associated with multiple renal stones $(p=0.053)$ and multiple tracts $(p=0.0579)$. However, lower calyx access ( $p=0.001)$, solitary kidney stones $(p=0.005)$, and multiple renal stones $(p=0.005)$ are independent prognostic factors of severe postoperative bleeding according to the multivariate analysis (Tables I and II).

\section{Discussion}

Percutaneous nephrolithotomy has replaced open surgery as the treatment of choice for most renal calculi, particularly staghorn stones [12]. The 
A
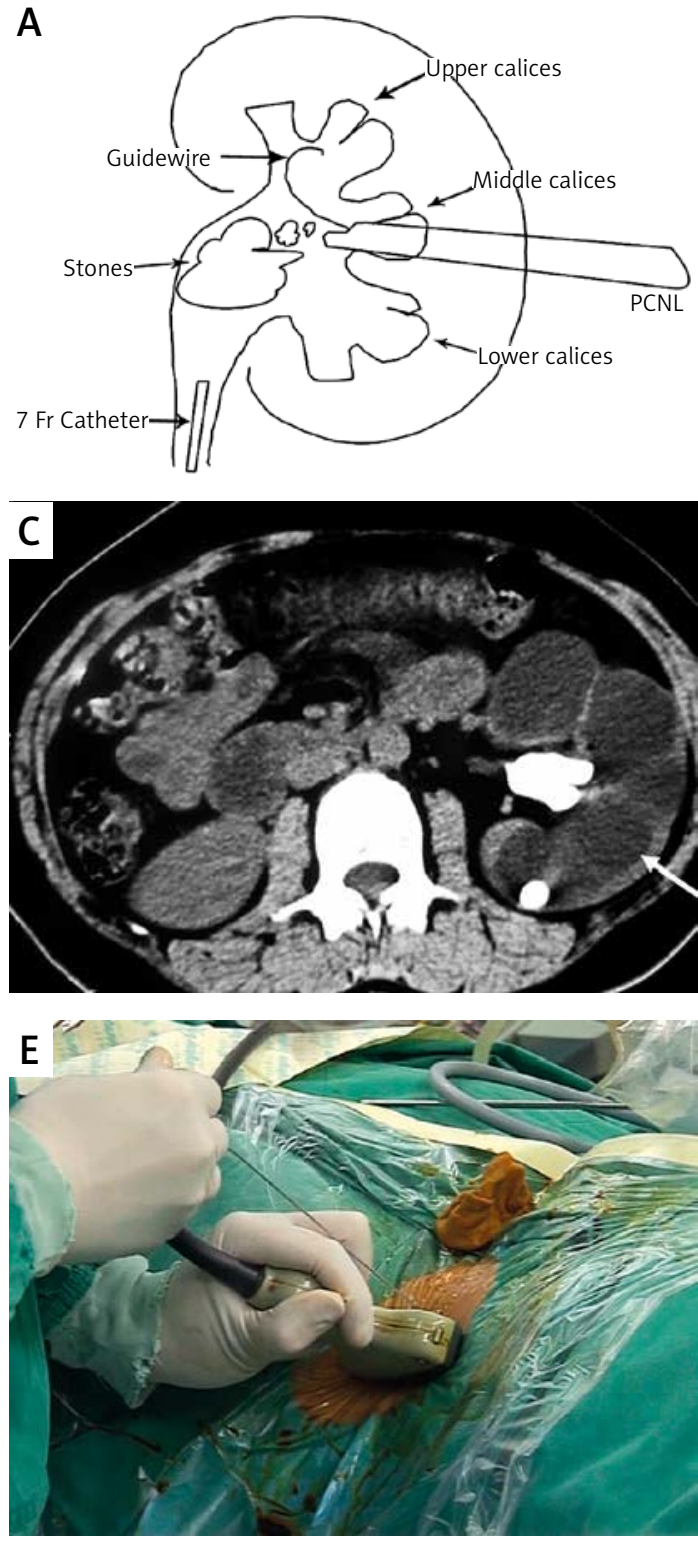

B
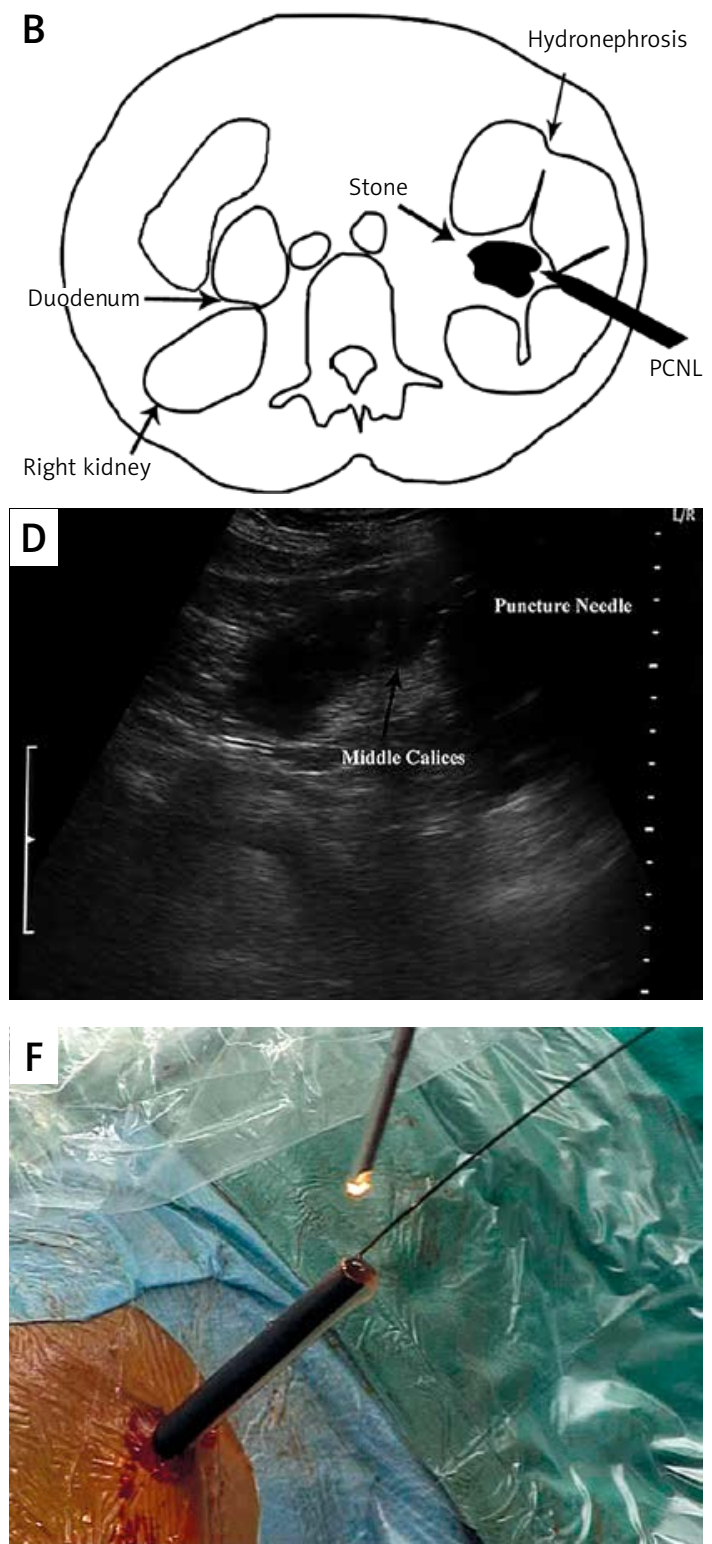

Figure 1. Percutaneous nephrolithotomy (PCNL) for patients with kidney stones. A - PCNL coronal view; B - PCNL transversal view; C - Puncture site, transversal CT scan of the upper abdomen showed one stone in each of the left renal pelvis and the calyces, the former causing increased volume and hydronephrosis of the left kidney; D - Intraoperative ultrasonography, under the guidance of ultrasonography, the upper arrow shows the end of the needle with strong echogenicity. The lower arrow shows the penetration site, where the hypoechoic area on the left is the lower calyces of the left kidney, and the hypoechoic area on the right the upper calyces of the left kidney. The bilateral shadow beneath the lower calyces of the left kidney represents the retrograde placement of the $7 \mathrm{Fr}$ ureteral catheter; $\mathbf{E}$ - With the patient prone, the penetration site was ultrasonically confirmed as the posterior calyceal fornix of the median calyces in the right kidney, allowing the maximum depletion of stones; $\mathbf{F}$ - With the patient prone and the operation channel established for percutaneous stone removal, the nephroscope was placed under the guidance of the safety wire. The entire renal pelvis was observed, and stones were crushed using the holmium laser and washed out through the operation channel with saline;

results from our study indicated that severe postoperative bleeding is associated with inferior calyx puncture access, multiple renal stones and isolated kidney stones. Renal parenchyma next to the lower pole calyx is rich in arterioles. Inferior calyx access requires an oblique and longer surgical tract. To reach the renal pelvis, the nephroscope often needs to be adjusted. The risk of laceration of renal parenchyma is therefore increased. Also, the lower calyx is smaller; accidental injury to the opposite side of the lower pole calyx is thus more likely.

Upper calyx access is associated with increased risk of intrathoracic complications [7, 13, 14]. We treated 85 cases with single upper calyx stones through upper calyx access with ultrasound guidance; hydrothorax or hemothorax was not noticed in any patient. Middle calyx access has been recommended for PCNL. The tract through middle 

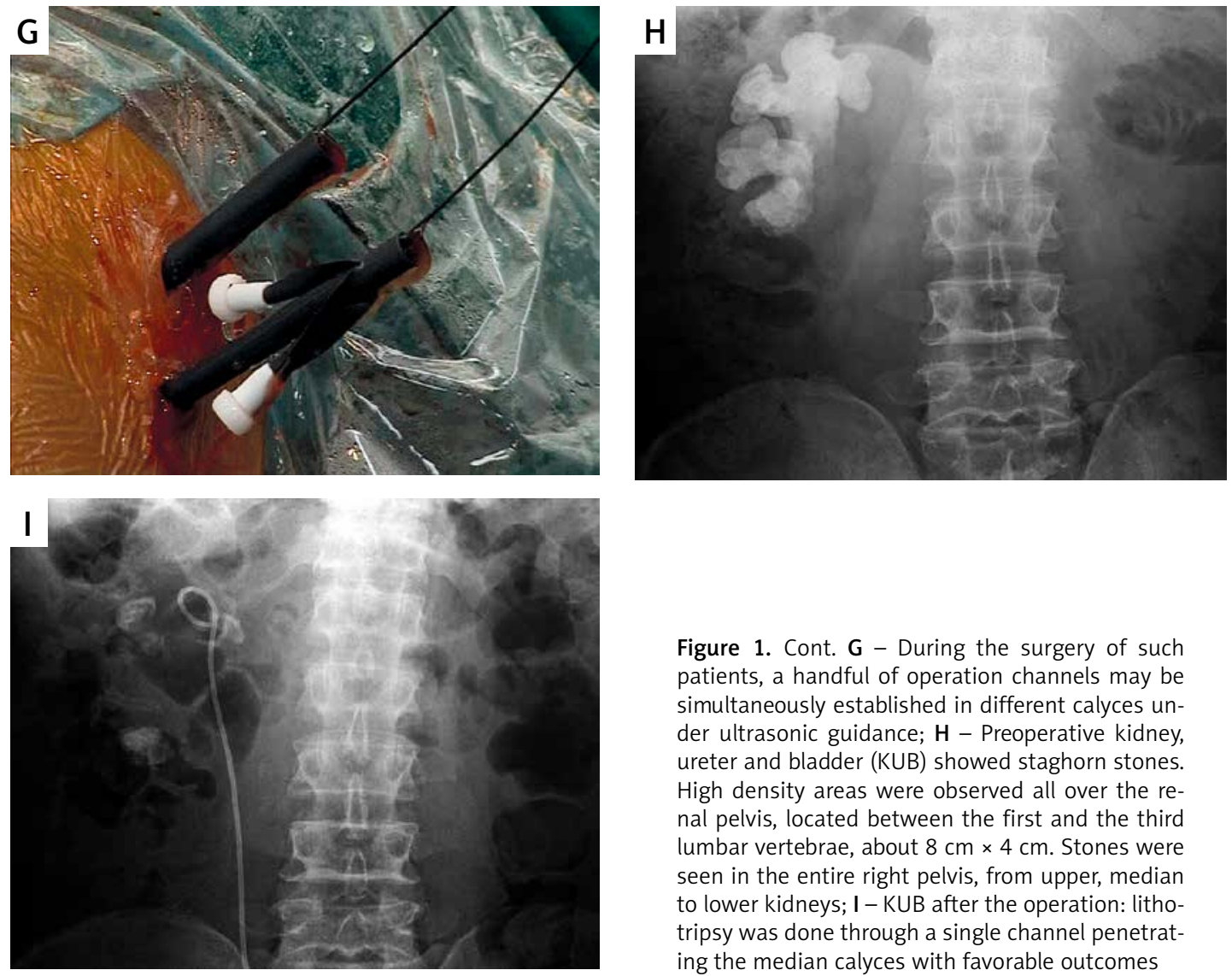

Figure 1. Cont. G - During the surgery of such patients, a handful of operation channels may be simultaneously established in different calyces under ultrasonic guidance; $\mathbf{H}$ - Preoperative kidney, ureter and bladder (KUB) showed staghorn stones. High density areas were observed all over the renal pelvis, located between the first and the third lumbar vertebrae, about $8 \mathrm{~cm} \times 4 \mathrm{~cm}$. Stones were seen in the entire right pelvis, from upper, median to lower kidneys; I - KUB after the operation: lithotripsy was done through a single channel penetrating the median calyces with favorable outcomes

Table I. Univariate analysis of the potential factors for severe postoperative bleeding

\begin{tabular}{|c|c|c|c|}
\hline Factors & $\begin{array}{l}\text { No severe postoperative } \\
\text { bleeding }(n=972)\end{array}$ & $\begin{array}{l}\text { Severe postoperative } \\
\text { bleeding }(n=10)\end{array}$ & Value of $p$ \\
\hline Stone type: & & & 0.053 \\
\hline Solitary stones & $469(48.3)$ & $2(20.0)$ & \\
\hline Multiple stones & $187(19.2)$ & $5(50.0)$ & \\
\hline Staghorn stones & $316(32.5)$ & $3(30.05)$ & \\
\hline Number of punctures & $2.17 \pm 1.31$ & $1.50 \pm 0.71$ & 0.125 \\
\hline Prior ESWL & $172(17.6)$ & $2(20.0)$ & 0.840 \\
\hline Multiple tracts & $121(12.4)$ & $1(10.0)$ & 0.0579 \\
\hline Puncture site (inferior calyx) & $238(24.5)$ & $8(80.0)$ & $<0.001$ \\
\hline $\begin{array}{l}\text { Solitary kidney or functional } \\
\text { solitary kidney stones }\end{array}$ & $135(13.9)$ & $4(40.0)$ & 0.030 \\
\hline Pre-existing urinary infection & $812(83.5)$ & $7(70.0)$ & 0.252 \\
\hline Operative time $[\mathrm{h}]$ & $1.62 \pm 0.73$ & $1.55 \pm 0.44$ & 0.748 \\
\hline $\begin{array}{l}\text { Chronic renal failure (serum } \\
\text { creatinine }>1.5 \mathrm{mg} / \mathrm{dl} \text { ) }\end{array}$ & 203 (20.9) & $2(20.0)$ & 0.945 \\
\hline
\end{tabular}

Results presented as $n(\%)$ or mean \pm standard deviation.

Table II. Multivariate logistic analysis of the potential factors for severe postoperative bleeding

\begin{tabular}{|lccccc|}
\hline \multirow{2}{*}{ Factors } & \multicolumn{2}{c}{ Characteristics } & Hazard ratio & 95\% Cl & \multirow{2}{*}{ Value of $p$} \\
\cline { 2 - 3 } & Unfavorable & Favorable & & & \\
\hline Site of puncture & Inferior calyx & Middle/upper calyx & 2.56 & $1.00-4.11$ & 0.001 \\
\hline Kidney stones & Solitary & Normal & 2.66 & $1.81-4.50$ & 0.005 \\
\hline Renal stones & Multiple & Single & 2.57 & $1.76-4.38$ & 0.005 \\
\hline
\end{tabular}


calyx access is shorter and manipulation of the stone is easier. In most cases, with the exception of renal infundibular stenoses, single middle calyx access is sufficient to remove the renal stones. Infundibular stenosis is typically the result of infection or scarring, and is characterized by a narrow infundibulum leading to a dilated or nondilated calyx with or without stone(s) [15]. In the current study, infundibular stenosis was noted in 24 out of 982 cases $(2.44 \%)$. We used holmium : YAG laser to open infundibular stenoses, but only achieved a success rate of $37.5 \%$ (9/24). Multi-channel puncture or ESWL was often needed to clear residual stones.

The incidence of severe postoperative bleeding in the current study is consistent with previous reports $[6,16,17]$ at approximately $1 \%$. In clinical practice in China, the diameter of tract dilation is $28 \mathrm{Fr}$ for conventional PCNL, and $20 \mathrm{Fr}$ for mini-PCNL. The operation involves several dilation stages and postoperative urine drainage through a nephrostomy tube. Severe postoperative bleeding was also associated with multiple renal stones and renal stones in solitary kidney. For renal stones in solitary kidney, compensatory hypertrophy of renal parenchyma could increase the risk of bleeding. According to related reports, retrograde flexible ureteroscopy may offer a safe and efficient modality for managing renal stones [18-25]. It is preferable to undergo retrograde ureteroscopy in selected patients with small stones in a solitary kidney who underwent neither SWL nor percutaneous stone removal. Severe postoperative bleeding may be correlated with multiple renal stones. The nephroscope often needs to be maneuvered through several calyceal necks. Selective renal angiography revealed injury to renal arterioles in all 10 cases with severe postoperative bleeding. A single treatment with selective embolization stopped the bleeding in 8 cases. In the remaining 2 cases, the bleeding was successfully controlled upon repeat embolization.

We are well aware that this is a retrospective study, and our results do not have the same strength as an observational study. However, it provides a substantial basis for the design of future randomized, prospective clinical trials and treatment strategies.

In conclusion, we can achieve satisfactory stone clearance rates through single middle calyx access in most cases except those with renal infundibular stenosis. Severe postoperative bleeding after PCNL is associated with renal puncture via the inferior calyx, multiple renal stones and solitary kidney stones.

\section{Conflict of interest}

The authors declare no conflict of interest.

\section{References}

1. Fernström I, Johansson B. Percutaneous pyelolithotomy. A new extraction technique. Scand J Urol Nephrol 1976; 10: 257-9.

2. Michel MS, Trojan L, Rassweiler JJ. Complications in percutaneous nephrolithotomy. Eur Urol 2007; 51: 899-906.

3. Skolarikos A, de la Rosette J. Prevention and treatment of complications following percutaneous nephrolithotomy. Curr Opin Urol 2008; 18: 229-34.

4. Patel SR, Haleblian GE, Pareek G. Percutaneous nephrolithotomy can be safely performed in the high-risk patient. Urology 2010; 75: 51-5.

5. Unsal A, Resorlu B, Atmaca AF, et al. Prediction of morbidity and mortality after percutaneous nephrolithotomy by using the Charlson Comorbidity Index. Urology 2012; 79: 55-60.

6. Kukreja R, Desai M, Patel S, Bapat S, Desai M. Factors affecting blood loss during percutaneous nephrolithotomy: prospective study. J Endourol 2004; 18: 715-22.

7. Nishizawa K, Yamada H, Miyazaki Y, Kobori G, Higashi Y. Results of treatment of renal calculi with lower-pole fluoroscopically guided percutaneous nephrolithotomy. Int J Urol 2008; 15: 399-402.

8. Unsal A, Resorlu B, Kara C, Bozkurt OF, Ozyuvali E. Safety and efficacy of percutaneous nephrolithotomy in infants, preschool age, and older children with different sizes of instruments. Urology 2010; 76: 247-52.

9. Ozden E, Mercimek MN, Yakupoğlu YK, Ozkaya O, Sarikaya S. Modified Clavien classification in percutaneous nephrolithotomy: assessment of complications in children. J Urol 2011; 185: 264-8.

10. Inoue Y, Yoshikawa K, Yoshioka N, et al. Evaluation of renal function with $99 \mathrm{mTC}-M A G 3$ using semiautomated regions of interest. J Nucl Med 2000; 41: 1947-54.

11. Chatham JR, Dykes TE, Kennon WG, Schwartz BF. Effect of percutaneous nephrolithotomy on differential renal function as measured by mercaptoacetyl triglycine nuclear renography. Urology 2002; 59: 522-5.

12. Preminger GM, Assimos DG, Lingeman JE, Nakada SY, Pearle MS, Wolf JS Jr; AUA Nephrolithiasis Guideline Panel). Chapter 1: AUA guideline on management of staghorn calculi: diagnosis and treatment recommendations. J Urol 2005; 173: 1991-2000.

13. Munver R, Delvecchio FC, Newman GE, Preminger GM. Critical analysis of supracostal access for percutaneous renal surgery. J Urol 2001; 166: 1242-6.

14. Narasimham DL, Jacobsson B, Vijayan P, Bhuyan BC, Nyman U, Holmquist B. Percutaneous nephrolithotomy through an intercostal approach. Acta Radiol 1991; 32: 162-5.

15. Kim HL, Gerber GS. Use of ureteroscopy and holmium:yttrium-aluminum-garnet laser in the treatment of an infundibular stenosis. Urology 2000; 55: 129-31.

16. Martin X, Murat FJ, Feitosa LC, et al. Severe bleeding after nephrolithotomy: results of hyperselective embolization. Eur Urol 2000; 37: 136-9.

17. El-Nahas AR, Shokeir AA, El-Assmy AM, et al. Post-percutaneous nephrolithotomy extensive hemorrhage: a study of risk factors. J Urol 2007; 177: 576-9.

18. Preminger GM. Management of lower pole renal calculi: shock wave lithotripsy versus percutaneous nephrolithotomy versus flexible ureteroscopy. Urol Res 2006; 34: 108-11.

19. Mariani AJ. Combined electrohydraulic and holmium:YAG laser ureteroscopic nephrolithotripsy of large (greater than $4 \mathrm{~cm}$ ) renal calculi. J Urol 2007; 177: 168-73.

20. Riley JM, Stearman L, Troxel S. Retrograde ureteroscopy for renal stones larger than $2.5 \mathrm{~cm}$. J Endourol 2009; 23: $1395-8$. 
21. Franczyk-Skóra B, Gluba A, Olszewski R, et al. Heart function disturbances in chronic kidney disease - echocardiographic indices. Arch Med Sci 2014; 10: 1109-16.

22. Khanagavi J, Gupta T, Aronow WS, et al. Hyperkalemia among hospitalized patients and association between duration of hyperkalemia and outcomes. Arch Med Sci 2014; 10: 251-7.

23. Kim MJ, Lee $H$, Kiang P, et al. Debunking the myth: low knowledge levels of HBV infection among Asian American college students. Asia-Pac J Oncol Nurs 2015; 2: 8-16.

24. Zhuo Y, Guo Q, Song P, et al. Correlation study and significance of the EGFR expression in serum, lymph nodes and tumor tissue of NSCLC. Thoracic Cancer 2014; 5: 31-7.

25. Fan Y, Yao Y, Li L, et al. nm23-H1 gene driven by hTERT promoter induces inhibition of invasive phenotype and metastasis of lung cancer xenograft in mice. Thoracic Cancer 2013; 4: 41-52. 